Jillian R. Tate*, David M. Bunk, Robert H. Christenson, Julian H. Barth, Alexey Katrukha, James E. Noble, Heinz Schimmel, Lili Wang and Mauro Panteghini, for the IFCC Working Group on Standardization of Cardiac Troponin I

\title{
Evaluation of standardization capability of current cardiac troponin I assays by a correlation study: results of an IFCC pilot project
}

DOI 10.1515/cclm-2014-1197

Received December 4, 2014; accepted December 17, 2014; previously published online February 19, 2015

\begin{abstract}
Background: As a part of an International Federation of Clinical Chemistry and Laboratory Medicine (IFCC) project to prepare a commutable reference material for cardiac troponin I (cTnI), a pilot study evaluated current cTnI assays for measurement equivalence and their standardization capability.
\end{abstract}

Methods: cTnI-positive samples collected from 90 patients with suspected acute myocardial infarction were assessed for method comparison by 16 cTnI commercial assays according to predefined testing protocols. Seven serum pools prepared from these samples were also assessed.

Results: Each assay was assessed against median cTnI concentrations measured by 16 cTnI assays using PassingBablok regression analysis of 79 patient samples with values above each assay's declared detection limit. We

\footnotetext{
*Corresponding author: Jillian R. Tate, Department of Chemical Pathology, Block 7 Floor 3, Royal Brisbane and Women's Hospital, Herston, 4029, Qld, Australia, Phone: +61 736460082 ,

Fax: +61 736463417 , E-mail: jill.tate@health.qld.gov.au David M. Bunk and Lili Wang: Material Measurement Laboratory, National Institute of Standards and Technology, Gaithersburg, MD, USA

Robert H. Christenson: Department of Pathology, University of Maryland School of Medicine, Baltimore, MD, USA

Julian H. Barth: Clinical Biochemistry, Leeds Teaching Hospitals NHS Trust Leeds General Infirmary, Leeds, UK

Alexey Katrukha: Research Department, HyTest Ltd, Turku, Finland James E. Noble: Analytical Science Group, National Physical Laboratory, Teddington, UK

Heinz Schimmel: European Commission, Joint Research Centre, Institute for Reference Materials and Measurements, Geel, Belgium Mauro Panteghini: Centre for Metrological Traceability in Laboratory Medicine (CIRME), University of Milan, Milan, Italy
}

observed a 10-fold difference in cTnI concentrations for lowest to highest measurement results. After mathematical recalibration of assays, the between-assay variation for patient samples reduced on average from $40 \%$ to $22 \%$ at low cTnI concentration, $37 \%-20 \%$ at medium concentration, and $29 \%-14 \%$ at high concentration. The average reduction for pools was larger at $16 \%, 13 \%$ and $7 \%$ for low, medium and high cTnI concentrations, respectively. Overall, assays demonstrated negligible bias after recalibration (y-intercept: -1.4 to $0.3 \mathrm{ng} / \mathrm{L}$ ); however, a few samples showed substantial positive and/or negative differences for individual cTnI assays.

Conclusions: All of the 16 commercial cTnI assays evaluated in the study demonstrated a significantly higher degree of measurement equivalence after mathematical recalibration, indicating that measurement harmonization or standardization would be effective at reducing inter-assay bias. Pooled sera behaved similarly to individual samples in most assays.

Keywords: cardiac troponin I; certified reference material; standardization.

\section{Introduction}

Cardiac troponins are critical biomarkers for the diagnosis of acute myocardial infarction (AMI) and for prognostic evaluation of patients with acute coronary syndrome (ACS) [1]. Accordingly, in the European Union in vitro diagnostic (IVD) medical devices classification, the importance of cardiac markers to patient healthcare and safety is highlighted [2]. The lack of standardized cardiac troponin I (cTnI) measurements for the many assays commercially available and consequent need to use assay-specific decision thresholds has the potential for confusion of physicians and misinterpretation of cTnI results; hence the urgency for cTnI standardization. 
cTnI assays are available from many IVD manufacturers and use different materials as calibrators and antibodies with various antigen epitope specificities. Consequently, cTnI results currently do not have metrological traceability to a higher-order reference [either through a reference measurement procedure and/or to a certified reference material (CRM)], but only traceability to different calibrators selected by each manufacturer [3]. A significant reason for non-equivalence of results among cTnI assays is likely to be the lack of a suitable CRM which all manufacturers could use to validate the accuracy of their assay calibration.

Over 10 years ago, the American Association for Clinical Chemistry (AACC) Troponin I Standardization Subcommittee attempted to standardize cTnI measurements for contemporary assays via the use of the troponin standard reference material (SRM) 2921 [4]. This SRM is composed of purified human troponin ternary complex, extracted from human heart, for which the cTnI concentration was determined through a combination of reversed-phase-liquid chromatography and amino acid analysis [5]. Studies performed using SRM 2921 showed that, when diluted in several diluents including pooled human serum this material did not behave in the same way as patient samples for the majority of routine cTnI assays available at the time of the study. After assay recalibration using the diluted SRM, approximately $50 \%$ of assays were still not in agreement [6]. The conclusion was that the proportion of routine assays demonstrating commutability for the SRM cTnI material was too low to successfully use this material for assay standardization by manufacturers.

An alternate proposed approach to achieving equivalence of cTnI measurements is to provide a matrixed CRM with demonstrated commutability with patient samples and routine cTnI assays [7-9]. This approach, for which a commutable CRM is available, has been applied to the standardization of various proteins, including quite recently cystatin C [10]. Several studies have shown that serum-based cTnI-positive materials could be used in assay calibration to reduce the difference in cTnI results between routine assays $[6,11,12]$. In the AACC study, result equivalence was observed after the mathematical realignment of values against the median $\mathrm{cTnI}$ concentrations of six pools, the variability of results among cTnI assays decreasing from CVs of approximately $90 \%$ to CVs of $7 \%-28 \%$ [6].

With these premises, the International Federation of Clinical Chemistry and Laboratory Medicine Working Group on Standardization of cTnI (IFCC WG-TNI) has undertaken a pilot project to investigate the feasibility of preparing a commutable, stable matrixed reference material for cTnI. The development of a matrixed (i.e., pooled-serum) reference material(s) to standardize/ harmonize cTnI assay measurement results will involve several steps, including the evaluation of the measurement equivalence of current cTnI assays, the commutability assessment of the candidate pooled-serum reference material(s), and the value-assignment of the commutable reference material(s). The WG-TNI pilot study aims to evaluate several of the development steps prior to the actual production of the reference material to mitigate the risks of producing a reference material for such a complex protein analyte. Key questions to be answered are:

1. Are current commercial assays capable of being harmonized/standardized using a mathematical recalibration to test for systematic differences?

2. Are pooled sera commutable with patient samples?

3. Can a pooled serum harmonize cTnI assays, i.e., produce equivalent measurement results within medically acceptable limits?

As an initial part of this study, 16 commercial assays were tested for measurement equivalence and the possibility for standardization. The prospects for future assay standardization are assessed by examining whether measurement results can first achieve equivalence within acceptable performance goals through a process of mathematical recalibration using a panel of individual patient sera. This is similar to the empirical harmonization approach for human thyroid stimulating hormone which compared commercial assays for systematic differences before and after mathematical recalibration [13]. The ability to mathematically recalibrate assays is an indication that the assays are measuring the same molecular form(s) of troponin that exist following myocardial tissue damage, despite the diversity of antibodies used. Assays that measure the same measurand can be, in theory, standardized if an appropriate reference material is available. The assessment of assay harmonization in our study, therefore, is done as a prelude to the development of a pooled serum CTnI CRM and is part of a larger goal towards standardization.

\section{Materials and methods}

\section{cTnl-positive patient samples}

Single samples were collected from 90 individual patients admitted (July 2010-September 2011) to the emergency department at the University of Maryland Medical Center in Baltimore, US with AMI and who were representative of those encountered in clinical practice. Blood was collected up to $72 \mathrm{~h}$ post-presentation. 
cTnI concentrations were required to be in the approximate range 50-20,000 ng/L [when measured by Advia Centaur Ultra-TnI ${ }^{\mathrm{TM}}$, Siemens Healthcare Diagnostics (Tarrytown, NY, USA)], with 30 samples required at three cTnI concentration levels, namely 50$500 \mathrm{ng} / \mathrm{L}$ (low “L” range), 500-5000 ng/L (medium “M” range) and 5000-20,000 ng/L (high "H” range).

The ethics approval for the study was obtained from the University of Baltimore, Maryland (RC, principal investigator) and the collecting laboratory obtained approximately $50 \mathrm{~mL}$ of blood from each consented patient. Blood was centrifuged for $15 \mathrm{~min}$ at $3000 \mathrm{~g}$, serum aliquoted within $4 \mathrm{~h}$ from collection and then stored frozen at $\leq-70^{\circ} \mathrm{C}$. Up to 30 aliquots of $0.5 \mathrm{~mL}$ and one aliquot of $3.5 \mathrm{~mL}$ or greater were prepared. Based on discussion with the assay manufacturers, serum was chosen as the study sample type.

\section{Serum pools}

Table 1 describes the serum pool preparation and their names. Seven cTnI-positive serum pools $(A-G)$ were prepared in an approximate cTnI concentration range 200-10,000 ng/L. A normal serum pool (NORM), used in the preparation of F and G cTnI pools, was obtained from a commercial blood collection centre (Bioreclamation, Baltimore, MD, USA; product number HMSRM-F) and blood was collected into Becton Dickinson (Franklin Lakes, NJ, USA) serum vacutainers. The WG-TNI stipulated that the blood be collected from 5 to 10 apparently healthy females aged $<30$ years, with body mass index $<25 \mathrm{~kg} /$ $\mathrm{m}^{2}$ and no reported history of heart disease. The NORM pool was filtered using a $0.2 \mu \mathrm{m}$ filter membrane. Next it was pre-screened for cTnI autoantibodies (cTnAAbs) using HyTest in-house assay that uses antibodies sensitive to cTnAAbs and that is similar to the assay described in [14]. Six cTnI-positive samples from AMI patients were diluted at 2:1, 1:1 and 1:2 in assay buffer (0.02 M Tris-HCl, pH 7.5 buffer, containing $0.15 \mathrm{M} \mathrm{KCl}, 0.005 \mathrm{M} \mathrm{CaCl}_{2}, 7.5 \% \mathrm{BSA}$ and $0.15 \% \mathrm{NaN}_{3}$ ) and in NORM pool, producing similar and higher recoveries of cTnI immunological activity compared with a cTnAAb-interference (IF) control sample from an apparently healthy donor with a high level of interfering factor (Supplementary File 1, Figure 1, that accompanies the article http://www.degruyter.com/view/j/cclm.2015.53.issue-6/ cclm-2014-1197/cclm-2014-1197.xml?format=INT).

Fresh-frozen materials (stored at $\leq-70^{\circ} \mathrm{C}$ ) were the best practical approach for this international study. Each participating laboratory received $90 \times 0.5 \mathrm{~mL}$ patient samples, pools $\mathrm{A}-\mathrm{G}$ (duplicate aliquots, vials 1 and 2), $2 \mathrm{~mL}$ of a pool prepared from high cTnI patient samples for interference testing and the NORM pool, which were shipped on dry ice to participating laboratories in November/December 2011. All patient samples and pools were stored frozen at $\leq-70{ }^{\circ} \mathrm{C}$ upon their receipt at the participating laboratory and prior to analysis to minimize any loss of sample integrity.

\section{Samples for checking system alignment and precision}

Up to three levels of a manufacturer's cTnI internal quality control material (IQC 1, 2 and 3) were requested to be assayed in duplicate at regular intervals during measurement of patient samples and pools.

\section{cTnl analytical systems}

Testing was carried out on the following systems from six diagnostic manufacturers: Abbott Architect i2000SR, Architect STAT, and AxSYM ADV (Abbott Diagnostics, Abbott Park, IL, USA), Beckman Access 2 (Beckman Coulter, Brea, CA, USA), Mitsubishi Pathfast and PathfastII (Mitsubishi Chemical Medience, Chiba, Japan), Ortho Vitros 5600 (Ortho Clinical Diagnostics, Rochester, NY, USA), Roche Elecsys cobas e411 and cobas e601 (Roche Diagnostics, Mannheim, Germany), Siemens Advia Centaur, Dimension EXL with LM, Dimension RxL, Dimension Vista, Immulite 1000 TPI, Immulite 2000/Xpi TPI, and Stratus CS (Siemens Healthcare Diagnostics, Tarrytown, NY, USA) [15].

\section{Testing protocols}

To optimize the study protocol, patient samples, pools and manufacturer's IQC were analyzed in three sample sets on Day 1 of the study in

Table 1 Name and preparation of cardiac troponin I (cTnl) serum pools.

\begin{tabular}{|c|c|}
\hline cTnl pool ID & Description \\
\hline Pool A & $\begin{array}{l}18 \text { low cTnl concentration patient samples pooled using volumes ranging from } 1.25 \mathrm{~mL} \text { to } 8.00 \mathrm{~mL} \text {. } \\
\text { Target cTnl concentration of pool } \approx 200 \mathrm{ng} / \mathrm{L} \text {. }\end{array}$ \\
\hline Pool B & $\begin{array}{l}21 \text { medium cTnl concentration patient samples pooled using volumes ranging from } 1.50 \mathrm{~mL} \text { to } 6.00 \mathrm{~mL} \text {. } \\
\text { Target cTnl concentration of pool } \approx 2000 \mathrm{ng} / \mathrm{L} \text {. }\end{array}$ \\
\hline Pool C & $\begin{array}{l}21 \text { high cTnl concentration patient samples pooled using volumes ranging from } 0.75 \mathrm{~mL} \text { to } 10.75 \mathrm{~mL} \text {. } \\
\text { Target cTnl concentration of pool } \approx 10,000 \mathrm{ng} / \mathrm{L} \text {. }\end{array}$ \\
\hline Pool D & $\begin{array}{l}\text { Blend of } 28.0 \mathrm{~mL} \text { pool A+7.0 mL pool C. } \\
\text { Target cTnl concentration of pool } \approx 2000 \mathrm{ng} / \mathrm{L} \text {. }\end{array}$ \\
\hline Pool E & $\begin{array}{l}\text { Blend of } 14.0 \mathrm{~mL} \text { pool } \mathrm{C}+21.0 \mathrm{~mL} \text { pool B. } \\
\text { Target cTnl concentration of pool } \approx 5000 \mathrm{ng} / \mathrm{L} \text {. }\end{array}$ \\
\hline Pool F & $\begin{array}{l}\text { Blend of } 4.0 \mathrm{~mL} \text { pool C }+36.0 \mathrm{~mL} \text { pool NORM. } \\
\text { Target cTnl concentration of pool } \approx 1000 \mathrm{ng} / \mathrm{L} \text {. }\end{array}$ \\
\hline Pool G & $\begin{array}{l}\text { Blend of } 4.0 \mathrm{~mL} \text { pool B+36.0 mL pool NORM. } \\
\text { Target cTnl concentration of pool } \approx 200 \mathrm{ng} / \mathrm{L} \text {. }\end{array}$ \\
\hline Pool NORM & Pool of 5-10 apparently healthy people (young females, $18-30$ years old) (purchased from commercial source). \\
\hline
\end{tabular}


a pre-specified order (Supplementary File 1). All samples, pools and IQC were analyzed in duplicate.

\section{Assay calibration}

Assays were calibrated according to the manufacturer's specifications and IQCs were to be within the manufacturer's assay recommended range prior to proceeding with analysis of the study samples.
Only one calibration was done unless IQCs were outside of manufacturer's specified limits.

\section{Sample preparation}

Sample preparation (thawing, mixing, and centrifugation) was staggered such that samples were not thawed for $>3 \mathrm{~h}$ prior to
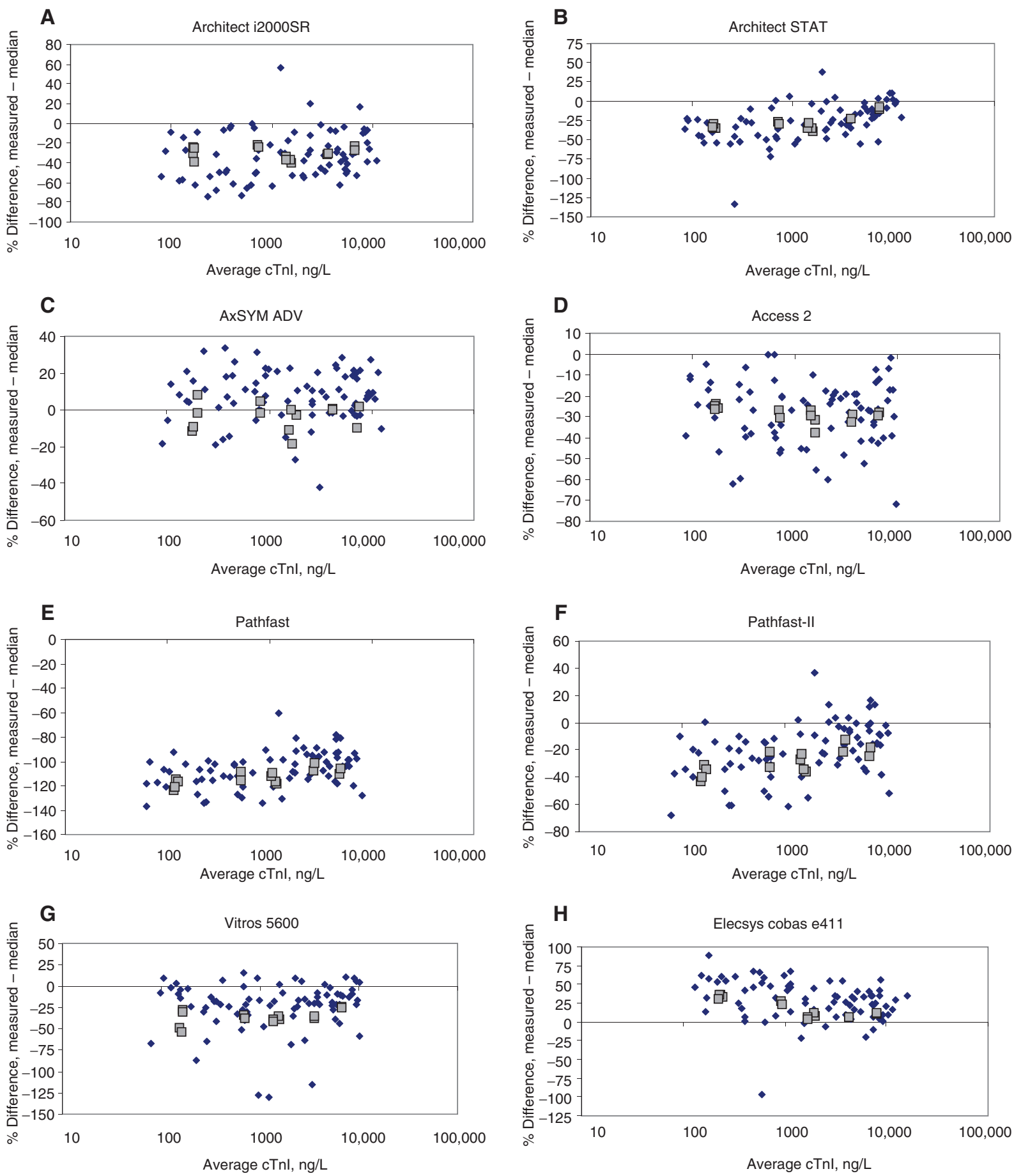

(Figure 1 Continued) 

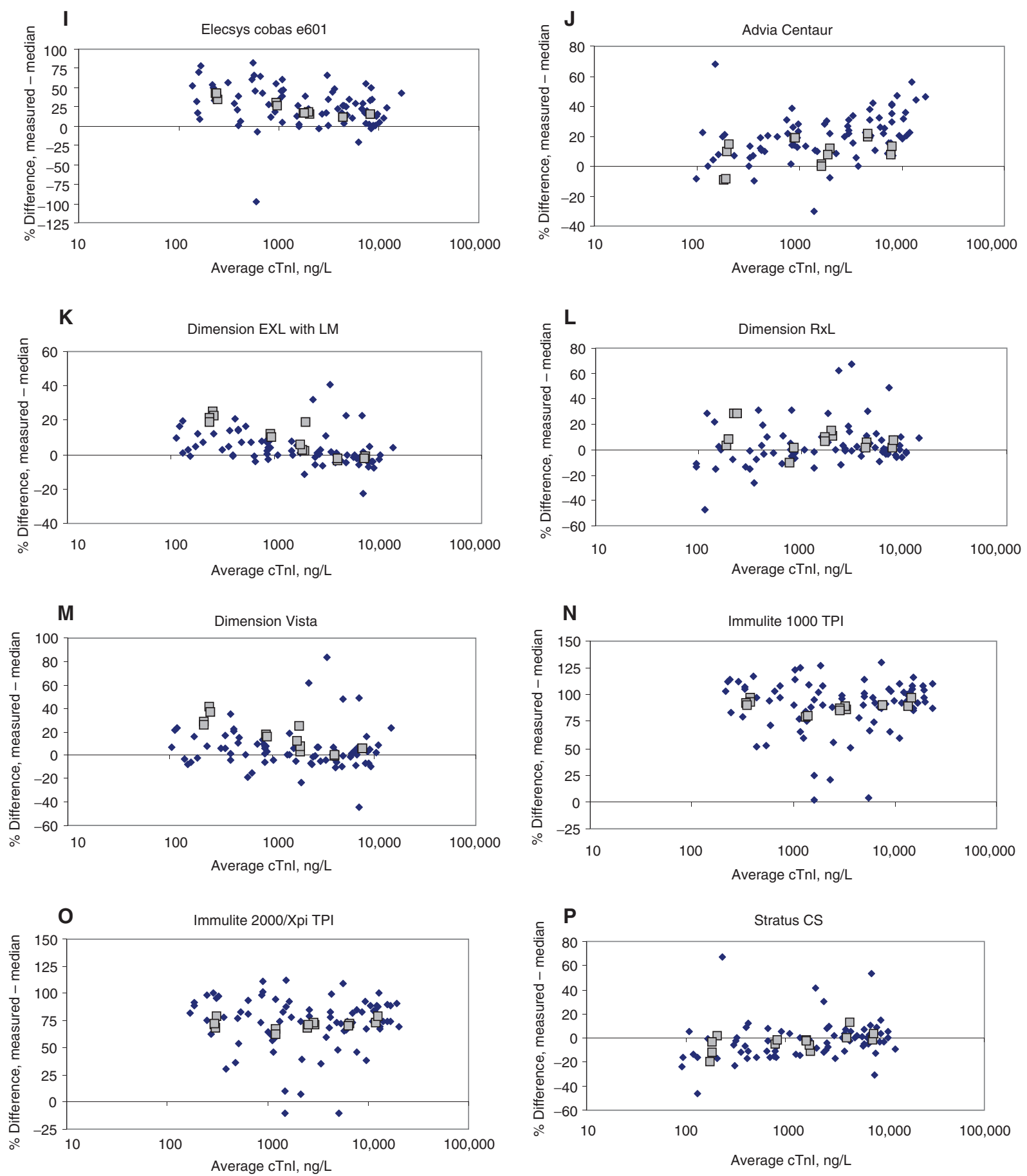

Figure 1 Difference plots in percentage for results of 79 patient samples (diamonds) and 7 pools (duplicate vials, squares) by 16 assays pre-recalibration.

completion of cTnI analysis and duplicate measurements of the same sample aliquot were not $>2 \mathrm{~h}$ apart. At the time of a run only the set of predetermined samples and pools to be analyzed were defrosted in a water bath (temperature $20 \pm 2{ }^{\circ} \mathrm{C}$ ) for $15 \mathrm{~min}$, hand mixed by inversion five times, then centrifuged according to the recommended sample preparation procedure of the manufacturer's package insert instructions.

\section{Working study protocol for method comparison - Day 1}

Two separate working study protocols (1 and 2) were developed to accommodate the sample throughput capabilities of the different assay systems to be used by participating laboratories, namely by automated analyzer (Protocol 1) and by Stratus CS (Protocol 2). To accommodate the number of samples to be analyzed on the one day, 
six Stratus analyzers were used. Excel spreadsheets for the two study protocols were provided for reporting measurement results. The working study protocols are detailed in Supplementary File 1.

\section{Working study protocol for interference testing - Day 2}

On Day 2, interference testing was performed. An aliquot of NORM was screened for interfering antibodies by participating laboratories using a dilution protocol to test for the presence of cTnAAbs and heterophilic antibody blocking tubes (Scantibodies, Santee, CA, USA) to test for heterophilic antibodies (HetAbs), the latter only if the cTnI result of NORM pool was above an assay's 99th percentile upper reference limit (99URL) (Supplementary File 1).

\section{Data analysis}

Within-assay precision was determined for each manufacturer's IQC from four duplicate measurements of IQC for 16 systems. Measurement precision of patient samples and serum pools was calculated as the $\mathrm{CV}$ for duplicate measurements of patient samples for each $\mathrm{cTnI}$ assay. The all methods CV was determined for the 16 methods that had complete data sets.

Eleven of the 90 individual patient samples were excluded from Passing-Bablok regression analysis due to incomplete data sets with one or more assays having undetectable values. All were low cTnI concentration samples namely, L1, L2, L5, L9, L12, L16, L21, L22, L23, L24, and L26. For each assay the average value was calculated for duplicate measurements of patient samples and pools then median values were determined for the 16 methods and used as the comparative value in regression analysis. The choices in data analysis are because some of the assay data appear to have non-parametric distributions of uncertainty across the study's measurement range (i.e., the use of Passing-Bablok regression) and because some of the serum samples were outliers for some assays and not others; the median is less sensitive to outliers than an average and was used to reduce the influence of non-parametric distributions of results.

cTnI values for each assay were corrected for calibration differences by a mathematical recalibration using a correction factor based on regression slope and y-intercept values for median values of 79 patient samples. Recalibrated cTnI equals ([measured cTnI minus y-intercept]/slope]).

Between-method variation for 16 assays was calculated from the median and standard estimate for cTnI values pre-recalibration, and from the mean and standard deviation for $\mathrm{cTnI}$ values post-recalibration. Difference values for each assay pre- and post-recalibration were calculated as (measured minus median) and (recalibrated minus median) cTnI concentration, respectively, and were plotted against the average of these values.

\section{Results}

\section{Sample integrity}

Turbidity, fibrin clots, and lipid layer were noted by some participating laboratories in 12 of the 90 patient serum samples and may have resulted in poor duplicate measurements by some assays. Of the 90 patient samples, 11 low cTnI concentration samples gave one or more results below an assay's limit of detection as declared by the manufacturer. These included nine by Elecsys cobas e601, seven by Elecsys cobas e411, four by Immulite 1000 TPI and five by Immulite 2000 Xpi/TPI (Supplementary File 1, Table 1).

In a separate analysis (Day 2 of the study), individual participating laboratories screened the NORM pool for presence of cTnAAbs and HetAbs, after an initial screening procedure had indicated there were no cTnAAbs present in the pool. In all assays, the cTnI concentration of the NORM pool was less than or equal to the manufacturer's reported limit of detection and none gave values above a manufacturer's quoted 99URL. Dilution of a high concentration cTnI pool in the NORM pool to $75 \%$, $50 \%$ and 25\% cTnI concentration levels did not result in under-recovery of cTnI by any of the 16 assays. These data demonstrate that cTnAAbs were not present (data not shown).

\section{Assay precision}

Assay precision was in agreement with each manufacturer's specifications for IQC (Supplementary File 1, Table 2). Within-run CVs for the duplicate cTnI measurement of patient samples and serum pools were generally $<10 \%$ for each of the 16 systems (Supplementary File 1, Table 1). In all, of $1594 \mathrm{cTnI}$ duplicate measurements, only 26 (3.3\%) gave a CV $>10 \%$. Serum pools had similar precision as individual patient samples over the tested cTnI concentration range (Supplementary File 1, Figure 2).

\section{Current status of $\mathrm{cTn}$ I measurement}

In the study measured results for each assay for the 79 patient samples were assessed against the median of cTnI concentrations by 16 systems using Passing-Bablok regression analysis. An approximate 10-fold difference in cTnI results for patient samples was observed among the 16 commercial assays for individual patient sample values over the concentration range tested (Figure 1A-P; Supplementary File 2). Pathfast gave the lowest cTnI values (regression slope, 0.331) and Immulite 1000 TPI the highest values (regression slope, 2.868) (Table 2; Supplementary File 1, Figure 3A-P). 
Table 2 Passing-Bablok regression analysis of measured cardiac troponin I (cTnl) values for 79 individual patient samples versus median

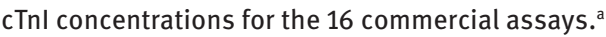

\begin{tabular}{lrr}
\hline Assay & Slope $(95 \% \mathrm{Cl})$ & Intercept, $\mathbf{n g} / \mathrm{L}(\mathbf{9 5} \% \mathrm{Cl})$ \\
\hline Abbott Architect i2000SR & $0.741(0.696-0.784)$ & $-21.8(-72$ to 17$)$ \\
Abbott Architect STAT & $0.859(0.816-0.911)$ & $-63.4(-124.2$ to -31.5$)$ \\
Abbott AxSYM ADV & $1.084(1.055-1.119)$ & $-5.3(-28.7$ to 24.9$)$ \\
Beckman Access 2 & $0.766(0.735-0.799)$ & $-0.8(-27.0$ to 22.0$)$ \\
Mitsubishi Pathfast & $0.331(0.317-0.346)$ & $-14.6(-34.3$ to -4.5$)$ \\
Mitsubishi Pathfast-II & $0.895(0.856-0.935)$ & $-41.4(-91.7$ to -16.0$)$ \\
OCD Vitros 5600 & $0.857(0.820-0.904)$ & $-21.5(-76.7$ to 7.2$)$ \\
Roche Elecsys cobas e411 & $1.261(1.181-1.352)$ & $79.3(7.3-141.7)$ \\
Roche Elecsys cobas e601 & $1.208(1.142-1.277)$ & $85.0(17.8-146.0)$ \\
Siemens Advia Centaur & $1.289(1.244-1.357)$ & $-44.2(-93.8$ to -15.1$)$ \\
Siemens Dimension EXL with LM & $0.959(0.938-0.989)$ & $61.9(31.6-113.9)$ \\
Siemens Dimension RxL & $1.004(0.989-1.025)$ & $-5.1(-27.1$ to 12.9$)$ \\
Siemens Dimension Vista & $1.020(0.992-1.045)$ & $10.2(-13.0$ to 28.5) \\
Siemens Immulite 1000 TPI & $2.868(2.722-3.054)$ & $-23.6(-310.5$ to 77.2$)$ \\
Siemens Immulite 2000/Xpi TPI & $2.257(2.152-2.427)$ & $7.2(-169.8$ to 81.9$)$ \\
Siemens Stratus CS & $1.002(0.975-1.021)$ & $-21.7(-51.8$ to 1.1$)$ \\
\hline
\end{tabular}

a11 samples (L1, L2, L5, L9, L12, L16, L21, L22, L23, L24, L26) were excluded from calculation due to one or more assays having undetectable values; L3 was also missing from Architect i2000SR and M10 from Elecsys cobas e411 and e601 analysis. Cl, confidence interval.

\section{Assay result equivalence after mathematical recalibration}

Following mathematical recalculation of cTnI results according to the regression findings reported in Table 2, the between-assay variation for cTnI results was reduced compared with uncorrected results. Prior to recalibration the mean variation (i.e., the $\mathrm{CV}$ ) for low $\mathrm{cTnI}$ concentration samples was $40 \%$ (range $11 \%-65 \%$ ) and following recalibration this reduced to a mean of $22 \%$ (range $11 \%-38 \%$ ) (Figure 2A). Note that after recalibration two samples (L10 and L19) still gave $>35 \%$ CV. For medium cTnI concentration samples, mean variation before and after recalibration was 37\% (range $16 \%-63 \%$ ) and $20 \%$ (range $7 \%-58 \%$ ), respectively (Figure 2B). Sample M14 after recalibration gave $>30 \%$ CV and samples M23, M25, and M27 gave $>50 \%$ $\mathrm{CV}$. The removal of these four samples reduced the mean variation after recalibration to $16 \%$ (range $7 \%-28 \%$ ). For high cTnI concentration samples, mean variation before and after recalibration was 29\% (range 13\%-63\%) and $14 \%$ (range $7 \%-42 \%$ ), respectively (Figure $2 \mathrm{C}$ ). After recalibration sample $\mathrm{H} 15$ gave $>40 \% \mathrm{CV}$. The removal of this outlier sample reduced the mean variation after recalibration to $13 \%$ (range $7 \%-24 \%$ ). In general, serum pools gave lower inter-assay variability after recalibration than the majority of patient samples, ranging from $7 \%$ for highest concentration pools C (7517 ng/L) and E (4155 ng/L), and from $11 \%$ to $15 \%$ for other pools (187-1845 ng/L), except for pool A at $196 \mathrm{ng} / \mathrm{L}$ cTnI median concentration which gave a CV of 18.5\% (Figure 2D).
Analysis of regression equations obtained with cTnI results after assay recalibration showed a very good agreement between results, with slopes between 0.999 and 1.001 and negligible intercepts in the range of -1.4 to $0.3 \mathrm{ng} / \mathrm{L}$ (Table 3; Supplementary File 1, Figure $4 \mathrm{~A}-\mathrm{P})$. Accordingly, difference plots showed minimal bias over the cTnI concentration range that was tested against the median of cTnI concentrations by 16 systems (Figure 3A-P). Some samples were still discordant between methods and gave large positive or negative biases ( $>50 \%$ ) for some cTnI assays (Table 3 ). Often the samples were discordant within a family of assays that used the same antibodies in the sandwich, i.e., Siemens Dimension series and Stratus CS, Roche Elecsys cobas, Siemens Immulite, Mitsubishi Pathfast, but not Abbott Architect and AxSYM platforms [16]. For example, Immulite assays showed a strong negative bias for samples M25, M26, M27 and H15, whereas the Dimension assays gave a markedly positive bias for samples M25 and M27 (Table 3). Thirteen different samples showed a deviation $> \pm 50 \%$ in one or more assays, with up to six assays affected by sample M27, three assays giving a marked negative difference and three a marked positive difference (Table 3).

The composition of pools A, B and C was such that no one sample was present at more than 10.75 weight $\%$ of the total pool's volume. Pools F and G were prepared by a further 10-fold dilution into the NORM pool of patient pools $\mathrm{C}$ and $\mathrm{B}$, respectively. Results from pooled samples, potentially containing one or more of the problematic 

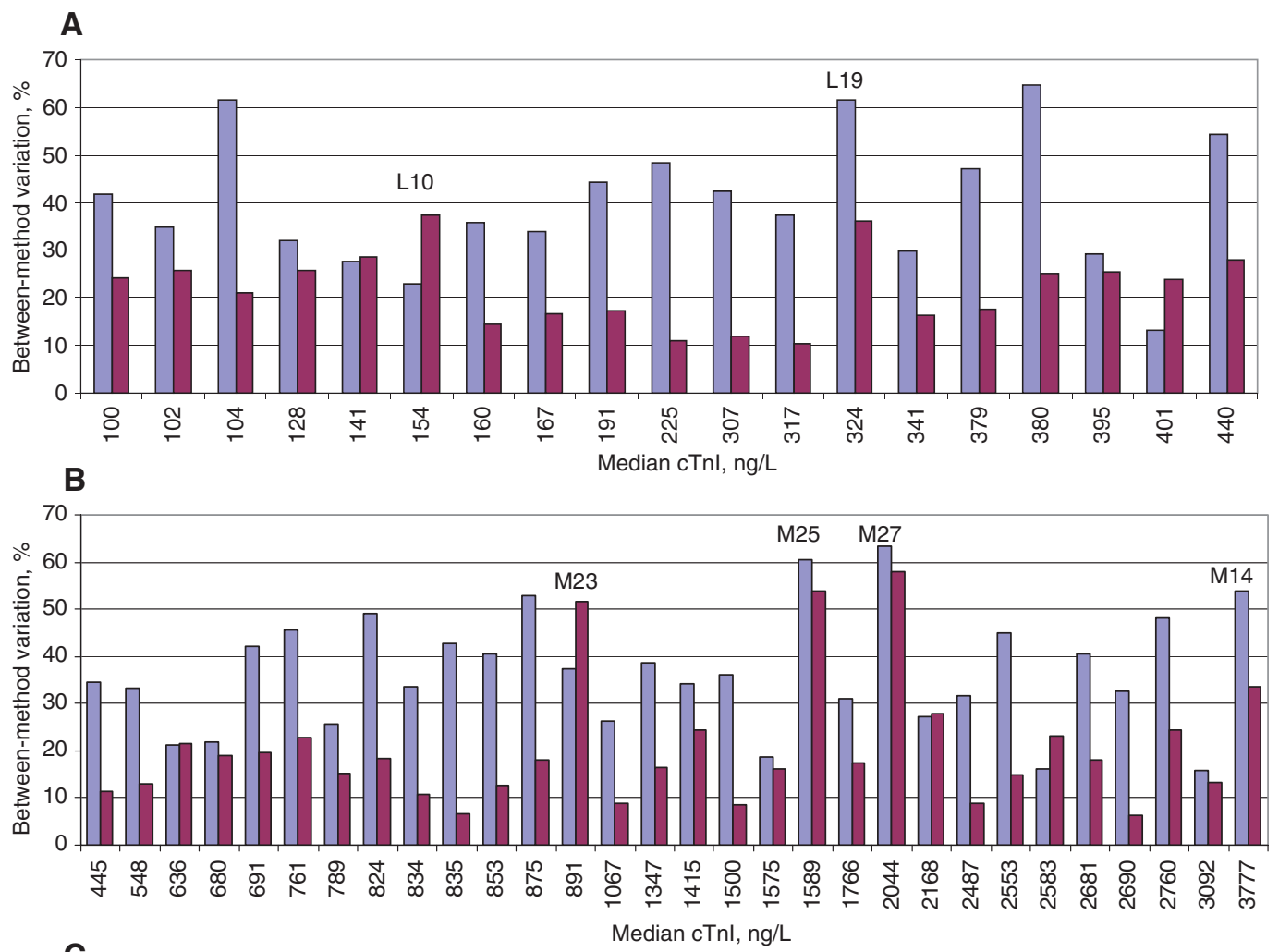

C
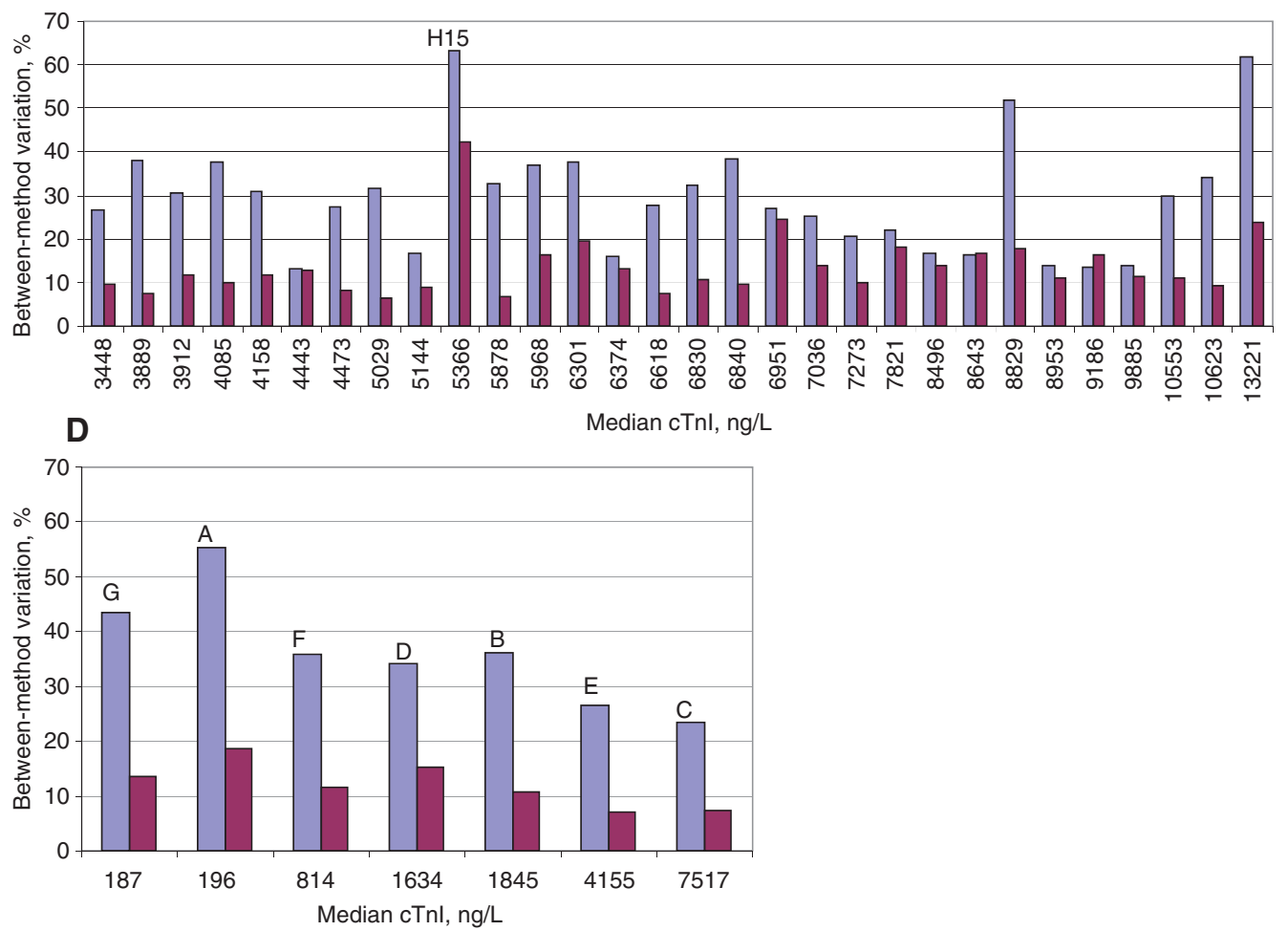

Figure 2 Between-assay variation of cTnl results from patient samples and serum pools before (gray columns) and after (red columns) mathematical recalibration of 16 systems.

(A) Between-assay variation of 19 low cTnl concentration samples. (B) Between-assay variation of 30 medium cTnl concentration samples.

(C) Between-assay variation of 30 high cTnl concentration samples. (D) Between-assay variation of $7 \mathrm{cTnl}$ serum pools, A-G. 
Table 3 Passing-Bablok regression analysis of post-recalibrated cardiac troponin I (cTnl) results in 79 patient samples for 16 evaluated assays versus median $\mathrm{CTnl}$ concentrations and discordant individual samples showing a difference $>50 \%$ post-recalibration. ${ }^{\mathrm{a}}$

\begin{tabular}{|c|c|c|c|}
\hline Assay & Slope $(95 \% \mathrm{Cl})$ & $\begin{array}{r}y \text {-intercept }(95 \% \mathrm{Cl}), \\
\mathrm{ng} / \mathrm{L}\end{array}$ & Discordant samples post-recalibration (Difference, in\%) \\
\hline Abbott Architect i2000SR & $1.000(0.940-1.057)$ & $0.3(-68.1$ to 51.8$)$ & M23 (84) \\
\hline Abbott Architect STAT & $1.000(0.950-1.061)$ & $-1.1(-70.8$ to -37.1$)$ & L15 (-86), M25 (55) \\
\hline Abbott AxSYM ADV & $0.999(0.973-1.033)$ & $-0.3(-22.1$ to 27.5$)$ & - \\
\hline Beckman Access 2 & $1.000(0.960-1.043)$ & $0.2(-34.2$ to 29.8$)$ & - \\
\hline Mitsubishi Pathfast & $1.001(0.957-1.046)$ & $-1.4(-60.9$ to 29.9$)$ & - \\
\hline Mitsubishi Pathfast-II & $1.001(0.956-1.044)$ & $-0.4(-56.8$ to 28.7$)$ & - \\
\hline OCD Vitros 5600 & $1.000(0.958-1.054)$ & $-0.5(-64.2$ to 33.3$)$ & L10 (-60), M2 (-53), M25 (-114), M27 (-118), H15 (-104) \\
\hline Roche Elecsys cobas e411 & $1.000(0.937-1.072)$ & $-0.1(-57.4$ to 49.4$)$ & $\operatorname{L} 19(-66), M 23(-132)$ \\
\hline Roche Elecsys cobas e601 & $1.000(0.945-1.057)$ & $-0.1(-56.1$ to 50.3$)$ & L3 (-51), L10 (56), L13 (-69), L19 (-76), M23 (-132) \\
\hline Siemens Advia Centaur & $1.000(0.965-1.052)$ & $-0.3(-38.3$ to 22.8$)$ & L11 (63), M25 (-51) \\
\hline $\begin{array}{l}\text { Siemens Dimension EXL with } \\
\text { LM }\end{array}$ & $1.000(0.978-1.031)$ & $-0.7(-31.5$ to 53.4$)$ & L3 (-58), M25 (66), M27 (84) \\
\hline Siemens Dimension RxL & $1.000(0.985-1.021)$ & $0.1(-21.5$ to 18.1$)$ & M25 (62), M27 (67) \\
\hline Siemens Dimension Vista & $1.000(0.973-1.024)$ & $0.3(-23.1$ to 17.6$)$ & M25 (60), M27 (82) \\
\hline Siemens Immulite 1000 TPI & $1.000(0.949-1.065)$ & $-0.4(-99.8$ to 36.3$)$ & M2 (-52), M25 (-94), M26 (-75), M27 (-79), H15 (-94) \\
\hline Siemens Immulite 2000/Xpi TPI & $1.000(0.954-1.075)$ & $0(-78.4$ to 32.8$)$ & L10 (-51), M25 (-87), M26 (-69), M27 (-72), H15 (-86) \\
\hline Siemens Stratus CS & $1.000(0.973-1.020)$ & $-0.4(-30.1$ to 22.6$)$ & L19 (73), H15 (54) \\
\hline
\end{tabular}

a11 samples (L1, L2, L5, L9, L12, L16, L21, L22, L23, L24, L26) were excluded from calculation due to one or more assays having undetectable values; L3 was also missing from Architect i2000SR and M10 from Elecsys cobas e411 and e601 analysis. Cl, confidence interval.

samples, were less affected and did not show sampledependent variation (Figure 3A-P).

\section{Discussion}

Efforts to standardize the measurement of the heterogeneous cTnI analyte began by defining the measurand as a unique, invariant part of the molecule that is common to all cTnI forms present in blood (i.e., amino acid sequence 30-110) and is present in the clinically relevant form(s) predominantly in AMI as a stable fragment of cTnI molecule complexed with cTnC [17]. Ideally, commercial immunoassay methods should recognize this particular sequence of amino acids common to all cTnI molecules present in blood, hence resulting in an increased equivalence of assay selectivity. In so doing, it is implied that all blood cTnI forms are measured by assays or the difference in reactivity is not clinically relevant. Standardization of cTnI assays requires, therefore, that differences in antibody specificities among the assays have no impact on assay results. Due to proteolytic susceptibility of N- and C-terminal parts of CTnI [18], the IFCC recommended several years back that diagnostic manufacturers use cTnI antibodies directed to the more stable mid-molecule epitopes [19] and not be affected by binary or ternary troponin complex formation and other 'in vivo' modifications $[9,19,20]$. Most of the currently available cTnI assays indeed use antibodies that are directed to the more stable mid-molecule epitopes, i.e., amino acids 30-110 [21]. As such, it should be possible to standardize the results from most routine cTnI assays.

From the experimental results of this study, it is apparent that some cTnI assays in the market give values that agree more closely than by other assays. Currently, we observed a 10 -fold absolute variation in cTnI results for lowest to highest assay measurement results. However, results for some manufacturers indicate that assays within a group of platforms are largely equivalent, i.e., Siemens's Dimension series and Stratus CS, Siemens Immulite 1000 and 2000/Xpi, Roche's Elecsys cobas e411 and e601 but not Siemens Advia Centaur, Abbott Architect and AxSYM, or Mitsubishi Pathfast and Pathfast II. The most important result of this study is the demonstration that systematic biases among cTnI assays can be successfully removed by an appropriate mathematical recalibration process with a residual inter-assay variation that falls within medically relevant limits. Although the definition of analytical performance goals for cTnI and cTnT measurements is still under discussion, based on biological variability $\left(\mathrm{CV}_{\text {intraindividual }} 9.7 \% ; \mathrm{CV}_{\text {interindividual }} 56.8 \%\right.$, obtained by $\mathrm{Wu}$ et al. [22]) a total CV $\leq 7.3 \%$ together with an assay bias 

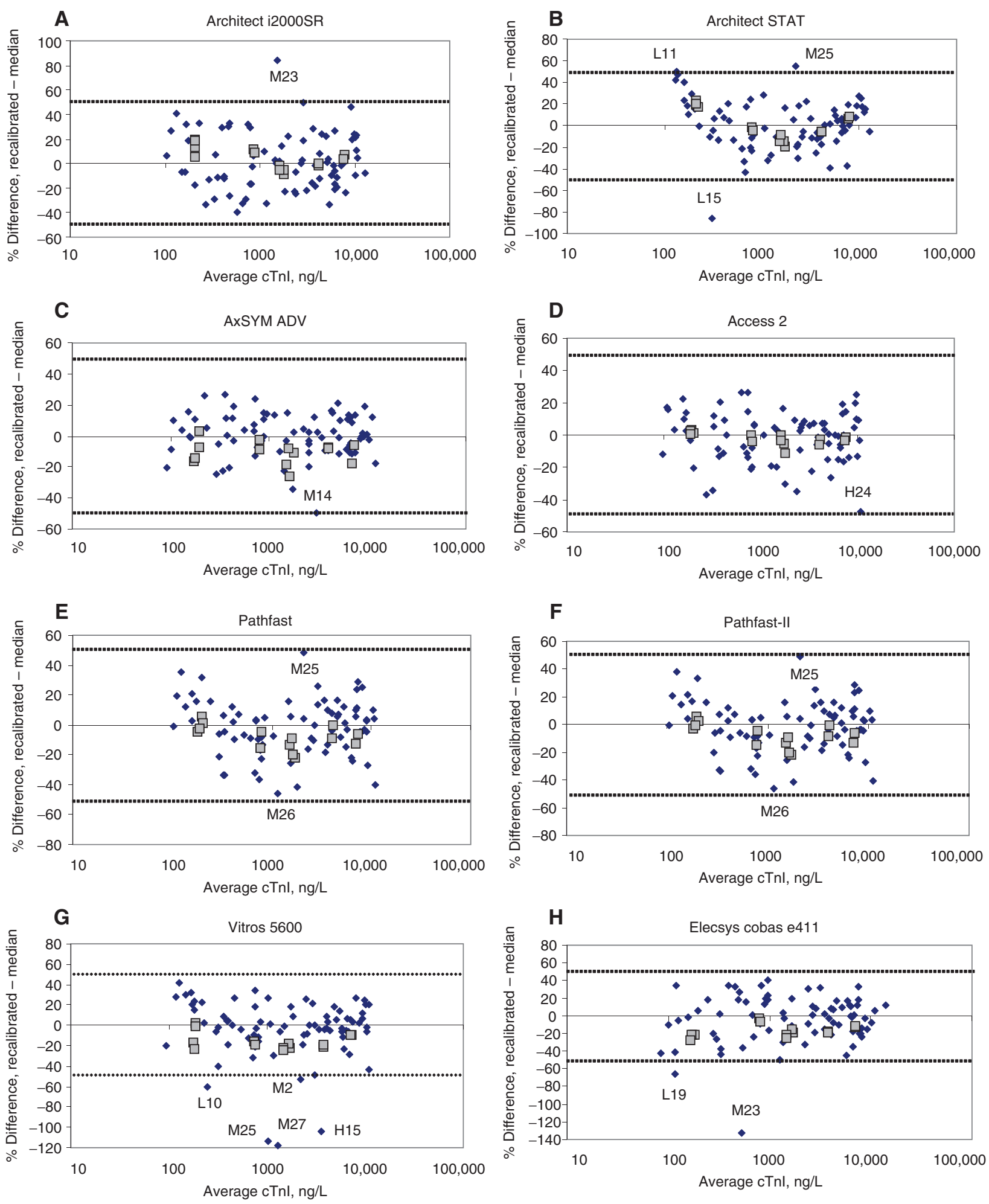

(Figure 3 Continued)

within $\pm 21.6 \%$ may reasonably represent a good compromise for minimum requirements [23]. This is consistent with the minimum total error goal for serum cTnI measurement estimated at $33.6 \%$ and is possible as shown in Figures 3A-P. However, it is clear that some cTnI assays showed a degree of positive or negative bias that increases at low concentration and which may contribute to poorer agreement of cTnI results. This observation 

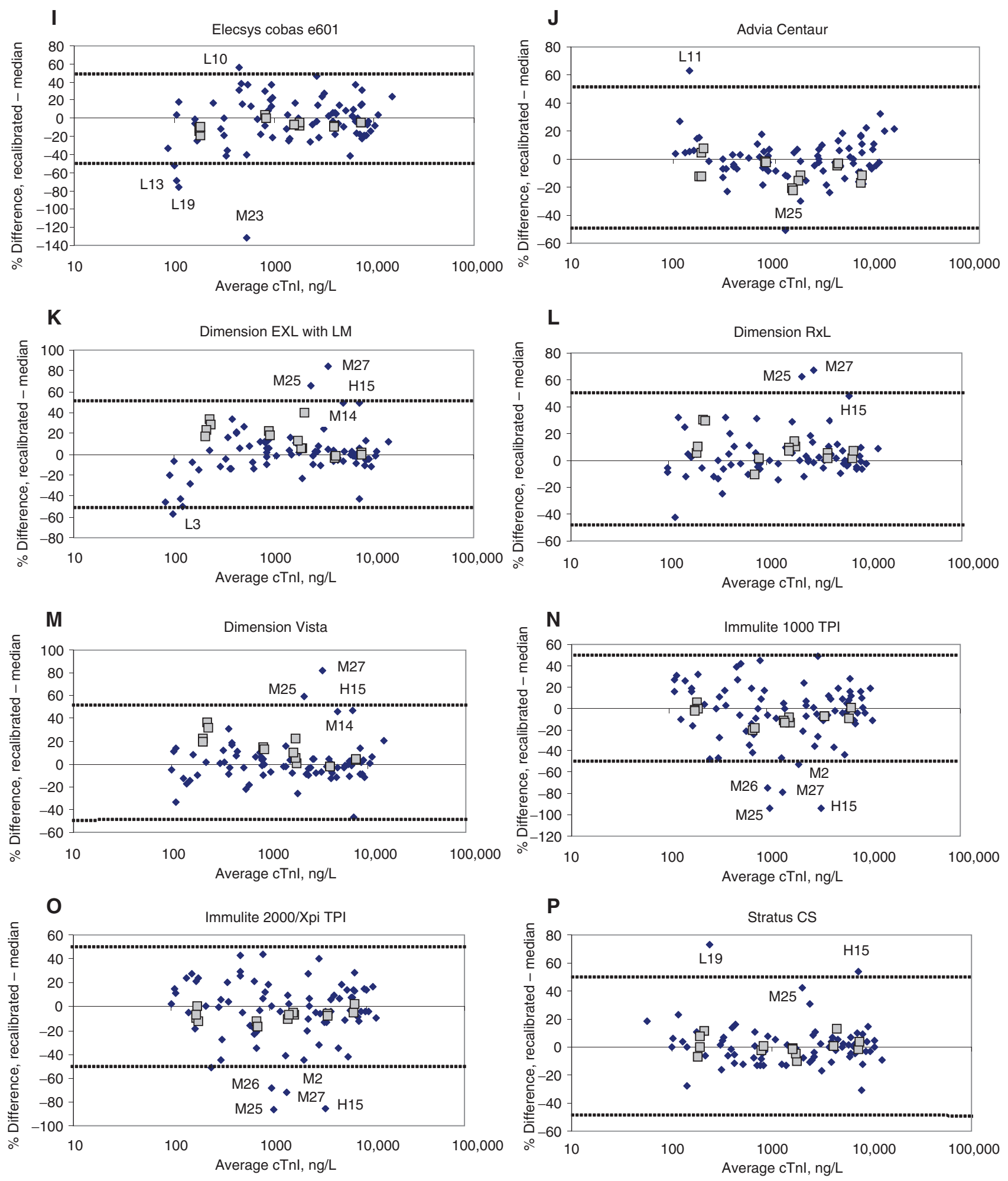

Figure 3 Difference plots in percentage for results of 79 patient samples (diamonds) and 7 pools (duplicate vials, squares) by 16 assays post-recalibration.

of "bias" of some assays as compared to the median may be due to the mismatch of the linear ranges of the assays used in the study. For some assays, the extremes of their measurement range are not always linear.
Our study also reports on the presence of sampledependent differences that occurred for individual manufacturers' assays and for series of assays with the same antibody specificity. Other studies have reported 
a sample-dependent variation that does occur for cTnI measurement probably reflecting differences in cTnI isoforms for some samples and different assay reactivity [12, 24]. Using cTnI assays available in the late 1990s Wu et al. showed that while assays recognized both the complexed and free cTnI forms, some did not give equal relative responses to the various forms of cTnI, hence resulting in over- or under-estimation of the cTnI concentration in patient samples [25]. In our study using a large number of samples from patients with suspected AMI/ACS, the sample-dependent effect appears largely related to antibody specificity as seen for the discordant results for the Dimension series of assays, as well as for each of the Immulite, Pathfast and Elecsys pairs of assays [16]. In the case of grossly discordant cTnI values that occurred for some samples including L19, M23, M25, M27, and H15 for which both over- and under-estimation of cTnI were observed in several assays some from different manufacturers, patient charts were reviewed by two clinicians. All of these patients were negative for stroke, cerebral ischemia, seizures, myocarditis, pulmonary infarction, celiac disease, renal failure, hypothyroidism, musculoskeletal diseases, viral infection or fever, connective tissue disorder and burns. If HetAbs or cTnAAbs are present, false cTnI values may occur [26-28]. Apart from testing the NORM pool for cTnAAbs and HetAbs by assessment of dilutional underrecovery of a high concentration CTnI pool in NORM pool or by an elevated value above an assay's limit of detection, of which none were observed for any of the 16 assays employed in the study, there was insufficient sample volume remaining to similarly test problematic patient samples further. Although fresh-frozen samples were used in this study, and provide the best practical material for such large multi-site evaluation studies, a huge underestimate from the median value in some assays due to a hypothetical degradation on thawing or structural configuration changes could be considered. However, with some exceptions, antibodies are all against the stable part of the molecule which ensures stability of the measurand [24]. Studies have shown that clinical samples stored frozen for several years at $-70{ }^{\circ} \mathrm{C}$ are stable $[29,30]$.

While systematic biases due to calibration differences between assays can be removed and agreement between assay results improved, as seen in this study, this does not mean that assays are uniform in their analytical and clinical performances. Consideration of assay characteristics, such as precision, bias, limit of detection, presence of interferences, etc. and of diagnostic accuracy using patient-based samples is also required.

Our study included several preparations of pooled serum with the goal of assessing their commutability to guide in the development of a future pooled serum CRM. The preparation of the pools varied from addition of individual patient sample sera at low, medium and high cTnI concentration (pools A, B and C), to admixtures of these pools (pools D and E), to dilution of pools B and C in NORM pool to produce pools $\mathrm{G}$ and F, respectively. The practical advantage of using diluted patient pools over undiluted ones is the larger volume of final reference material for cTnI which can be produced. According to the method correlation results these additional "biological" samples did behave like "native" patient samples in most assays. A fuller, statistically-based commutability assessment of the tested materials will be discussed in a future manuscript. Our study was not designed with the goal of using these pools for assay recalibration; a study for recalibration/standardization with pools would have included more replicate measurements of the pools and multiple dilutions of the pools in order to establish strong correlation data. While the current study was not carried out with these considerations, they will be incorporated into the WG-TNI's next effort to assign a value to a CRM based on the consensus of commercial assay measurement.

In conclusion, the results of the current study evaluating the equivalence of commercial cTnI assays and the means to preparing commutable serum pools provide the ground work for the future production and development of a pooled serum CRM for CTnI. By removing calibration differences between assays after mathematical recalibration all commercial assays have demonstrated a higher degree of measurement equivalence, indicating that measurement harmonization or standardization would be effective at reducing inter-assay bias.

Acknowledgments: We thank the following people and companies, who participated in this study: Jessie Shih, Tiffany Heilman, Robert Hruska, and Ryan Workman, Abbott Diagnostics, Abbott Park, IL; James Sackrison and John Branson, Beckman Coulter, Chaska, MN; Matsuya Takeshi, and Hiroyuki Yokoi, Mitsubishi Chemical Medience Corp., Chiba, Japan; Ralf Thomae, Mitsubishi Chemical Europe, Düsseldorf, Germany; Sheryl Sullivan, Ortho Clinical Diagnostics, Rochester, NY; Klaus Hallermayer, Roche Diagnostics, Penzberg, Germany; Roger Bauer, Gary Hickey, and Robert Payne, Siemens Healthcare Diagnostics, Newark, DE and Tarrytown, NY.

Disclaimer: Certain commercial equipment, instruments, and materials are identified in this manuscript to adequately specify the experimental procedures. Such identification does not imply recommendation or endorsement by NIST nor does it imply that the equipment, instruments, 
or materials are necessarily the best available for the purpose.

Author contributions: All the authors have accepted responsibility for the entire content of this submitted manuscript and approved submission.

Financial support: None declared.

Employment or leadership: None declared.

Honorarium: None declared.

Competing interests: The funding organization(s) played no role in the study design; in the collection, analysis, and interpretation of data; in the writing of the report; or in the decision to submit the report for publication.

\section{References}

1. Thygesen K, Alpert JS, Jaffe AS, Simoons ML, Chaitman BR, White HD: the Writing Group on behalf of the Joint ESC/ACCF/ AHA/WHF Task Force for the Universal Definition of Myocardial Infarction. Third Universal Definition of Myocardial Infarction. Circulation 2012;126:2020-35.

2. Global Harmonization Task Force (GHTF) Principles of IVD medical devices classification. Available from: http://www.imdrf.org/ docs/ghtf/archived/sg1/technical-docs/ghtf-sg1-n045r12-invitro-diagnostic-classification-070209.pdf. Accessed 13 March, 2014.

3. ISO 17511:2003: In vitro diagnostic medical devices - Measurement of quantities in biological samples - Metrological traceability of values assigned to calibrators and control materials.

4. Christenson RH, Duh SH, Apple FS, Bodor GS, Bunk DM, Dalluge J, et al. Standardization of cardiac troponin I assays: round robin of ten candidate reference materials. Clin Chem 2001;47:471-7.

5. Bunk DM, Welch MJ. Characterization of a new certified reference material for human cardiac troponin I. Clin Chem 2006;52:212-9.

6. Christenson RH, Duh SH, Apple FS, Bodor GS, Bunk DM, Dalluge J, et al. Towards standardization of cardiac troponin I measurements. Part II: Assessing commutability of candidate reference materials and harmonization of cardiac troponin I assays. Clin Chem 2006;52:1685-92.

7. Dati F, Panteghini M, Apple FS, Christenson RH, Mair J, Wu AH. Proposals from IFCC Committee on Standardization of Markers of Cardiac Damage (C-SMCD): strategies and concepts on standardization of cardiac marker assays. Scand J Clin Lab Invest 1999;59(Suppl 230):113-23.

8. Panteghini M. Current concepts in standardization of cardiac marker immunoassays. Clin Chem Lab Med 2004;42: 3-8.

9. Panteghini M. Standardization of cardiac troponin I measurements: the way forward? Clin Chem 2005;51:1594-7.

10. Zegers I, Keller T, Schreiber W, Sheldon J, Albertini R, BlirupJensen $\mathrm{S}$, et al. Characterization of the new serum protein reference material ERM-DA470k/IFCC: value assignment by immunoassay. Clin Chem 2010;56:1880-8.
11. Katrukha A, Bereznikova A, Pettersson K. New approach to standardization of human cardiac troponin I (cTnl). Scand I Clin Lab Invest 1999;59(Suppl 230):124-7.

12. Tate JR, Heathcote D, Koerbin G, Thean G, Andriske D, Bonar J, et al. The harmonization of cardiac troponin I measurement is independent of sample time collection but is dependent on the source of calibrator. Clin Chim Acta 2002;324:13-23.

13. Thienpont LM, Van Uytfanghe K, Beastall G, Faix JD, leiri T, Miller WG, et al. Report of the IFCC Working Group for Standardization of Thyroid Function Tests; Part 1: Thyroid-stimulating hormone. Clin Chem 2010;56:902-11.

14. Eriksson S, Ilva T, Becker C, Lund J, Porela P, Pulkki K, et al. Comparison of cardiac troponin I immunoassays variably affected by circulating autoantibodies. Clin Chem 2005;51:848-55.

15. Tate JR. Troponin revisited 2008: assay performance. Clin Chem Lab Med 2008;46:1489-500.

16. IFCC Troponin table. Available from: http://www.ifcc.org/ media/245202/IFCC\%20Troponin\%20I\%20and\%20T\%20 \%28ng_L\%20units\%29_\%20update\%20December\%202013. pdf; Accessed January, 2014.

17. Panteghini M. Selection of antibodies and epitopes for cardiac troponin immunoassays: should we revise our evidence-based beliefs? [editorial]. Clin Chem 2005;51:803-4.

18. Katrukha AG, Bereznikova AV, Filatov VL, Esakova TV, Kolosova OV, Pettersson K, et al. Degradation of cardiac troponin I: implication for reliable immunodetection. Clin Chem 1998;44:2433-40.

19. Panteghini M, Gerhardt W, Apple FS, Dati F, Ravkilde J, Wu AH. Quality specifications for cardiac troponin assays. International Federation of Clinical Chemistry and Laboratory Medicine (IFCC). Clin Chem Lab Med 2001;39:174-8.

20. Katrukha AG, Bereznikova AV, Esakova TV, Petterson K, Lövgren T, Severina ME, et al. Troponin I is released in bloodstream of patients with acute myocardial infarction not in free form but as complex. Clin Chem 1997;43:1379-85.

21. Panteghini M. Assay-related issues in the measurement of cardiac troponins. Clin Chim Acta 2009;402:88-93.

22. Wu AH, Lu AQ, Todd J, Moecks J, Wians F. Short- and long-term biological variation in cardiac troponin I measured with a highsensitivity assay: implications for clinical practice. Clin Chem 2009;55:52-8.

23. Panteghini M. Quality requirements for troponin assays - an overview. In: Laboratory and clinical issues affecting the measurement and reporting of cardiac troponins: a guide for clinical laboratories, 2012, The Australasian Association of Clinical Biochemists Inc., 2012:53-61.

24. Ungerer JP, Marquart L, O’Rourke PK, Wilgen U, Pretorius CJ. Concordance, variance, and outliers in 4 contemporary cardiac troponin assays: implications for harmonization. Clin Chem 2012;58:274-83.

25. Wu AH, Feng YJ, Moore R, Apple FS, McPherson PH, Buechler $\mathrm{KF}$, et al. Characterization of cardiac troponin subunit release into serum after acute myocardial infarction and comparison of assays for troponin T and I. Clin Chem 1998;44:1198-208.

26. Eriksson S, Halenius H, Pulkki K, Hellman J, Pettersson K. Negative interference in cardiac troponin I immunoassays by circulating troponin autoantibodies. Clin Chem 2005;51:839-47.

27. Adamczyk M, Brashear RJ, Mattingly PG. Circulating cardiac troponin-I autoantibodies in human plasma and serum. Ann N Y Acad Sci 2009;1173:67-74. 
28. Pettersson K, Eriksson S, Wittfooth S, Engström E, Nieminen M, Sinisalo J. Autoantibodies to cardiac troponin associate with higher initial concentrations and longer release of troponin I in acute coronary syndrome patients. Clin Chem 2009;55:

938-45.

29. Eggers KM, Jaffe $S$, Lind L, Venge P, Lindahl B. Value of cardiac troponin I cutoff concentrations below the 99th percentile for clinical decision-making. Clin Chem 2009;55:85-92.
30. Venge P, Lindahl B. Cardiac troponin assay classification by both clinical and analytical performance characteristics: A study on outcome prediction. Clin Chem 2013;59:976-81.

Supplemental Material: The online version of this article (DOI: 10.1515/cclm-2014-1197) offers supplementary material, available to authorized users. 